\title{
Vorbemerkung der Herausgeberinnen und Herausgeber
}

\author{
Klaus Beck • Christiane Eilders · Ines Engelmann · Christian Pentzold
}

Angenommen: 11. August 2021 / Online publiziert: 3. September 2021

(C) Der/die Autor(en) 2021

Rudolf Stöbers Beitrag „Genderstern und Binnen-I. Zu falscher Symbolpolitik in Zeiten eines zunehmenden Illiberalismus" in Heft 1 hat zahlreiche Reaktionen ausgelöst. Der Offene Brief an den DGPuK-Vorstand, zu dem wir bereits in Heft 1 sowie auf der DGPuK-Website Stellung genommen haben, die Debatte im DGPuKOnlineforum und die zahlreichen Repliken, die wir gerne abgedruckt haben, zeigen: Über die aus unterschiedlichen Gründen geäußerte Empörung hinaus gibt es eine ganze Reihe von fachlichen Argumenten, die der Debatte wert sind. In der vorliegenden Ausgabe schließen wir diese Debatte im Meinungsforum der Publizistik mit einer Stellungnahme von Kolleg*innen der FG Medien und Sprache ab, und wir räumen Rudolf Stöber die Möglichkeit ein, auf diese Repliken noch einmal kurz zu antworten.

Aus unserer Sicht hat die kontroverse Diskussion mindestens zwei Dinge sehr deutlich werden lassen: Zum einen handelt es sich beim Thema ,Gendern von Sprache" nicht nur um ein gesellschaftspolitisch relevantes Thema, sondern um ein sprach- wie kommunikationswissenschaftlich relevantes Forschungsfeld. Wie der

Prof. Dr. Klaus Beck $(\varangle)$

Fachbereich Kommunikationswissenschaft, Universität Greifswald,

Ernst-Lohmeyer-Platz 3, 17487 Greifswald, Deutschland

E-Mail: klaus.beck@uni-greifswald.de

Prof. Dr. Christiane Eilders

Düsseldorf, Deutschland

E-Mail: christiane.eilders@phil.uni-duesseldorf.de

Prof. Dr. Ines Engelmann

Jena, Deutschland

E-Mail: ines.engelmann@uni-jena.de

Prof. Dr. Christian Pentzold

Leipzig, Deutschland

E-Mail: christian.pentzold@uni-leipzig.de 
Beitrag aus der FG Medien und Sprache belegt, lohnt eine differenziertere Auseinandersetzung mit den Befunden in diesem interdisziplinären Feld. Ein Blick auf die über die DGPuK-Website zur Verfügung gestellte Literaturempfehlungsliste zeigt, wie sinnvoll und notwendig empirische Forschung aus dezidiert kommunikationswissenschaftlicher Perspektive ist, um die primär linguistisch basierte Forschungslage zu verbreitern. Wir möchten diesen fachwissenschaftlichen Diskurs gerne in der Publizistik wie in der Vergangenheit auch künftig befördern und laden ausdrücklich dazu ein, theoretisch und empirisch fundierte kommunikationswissenschaftliche Beiträge zur Publikation unter Peer-Review-Bedingungen einzureichen. In dieser Ausgabe finden Sie bereits einen solchen empirisch fundierten Beitrag von Sven Jöckel, Leyla Dogruel und Ronja Bachofer zur Wirkung gendergerechter Anmoderationen.

Zum anderen hat die Kontroverse gezeigt, dass in unserem Fach unterschiedliche Auffassungen über die Schranken bzw. Grenzen der Kommunikations- und Wissenschaftsfreiheit bestehen. Dabei geht es nicht nur um die Frage, ob beispielsweise universitäre oder redaktionelle Vorgaben zur gendergerechten Sprachverwendung diese Grundfreiheiten beschränken und ob es hierfür legitime Gründe gibt. Es geht auch um die Frage des Meinungsdiskurses in der Wissenschaft, konkret: in wissenschaftlichen Fachzeitschriften wie der Publizistik. Im Offenen Brief und einigen Beiträgen des DGPuK-Forums wurde der Publizistik „,redaktionelles Versagen“ und den Herausgeber*innen die Bewirtschaftung von Empörungskultur vorgeworfen. Es wurde die These aufgestellt, Kommunikationsfreiheit bzw. Meinungsfreiheit diene der Publizistik und einigen, die sich in der Debatte zu Wort gemeldet hatten, als Deckmantel oder „Strohmannargument“ zur Verstärkung von Meinungen ohnehin privilegierter professoraler Sprecher (hier nicht im generischen Maskulinum).

Diese Fragen scheinen uns als Herausgeber*innen und Redaktion diskussionswürdig. Wir möchten deshalb eine Debatte über die Kommunikations- und Meinungsfreiheit - auch, aber nicht nur in wissenschaftlichen Fachzeitschriften - und die legitimen Schranken dieser Freiheiten anregen. Die medien- bzw. presserechtliche Seite scheint mit Blick auf die Repliken und Debatten wenig strittig (aber auch hierzu würden uns andere Meinungen selbstverständlich interessieren). Für relevanter halten wir die kommunikations- und medienethische Seite: Einige der Diskussionsbeiträge führen ja (diskurs)ethische Kriterien und Gebote als Argumente an, allerdings mit recht unterschiedlichem Ergebnis. Wir haben zunächst zwei Kolleg*innen eingeladen, in der nächsten Ausgabe aus medienethischer Perspektive über die (legitimen) Grenzen der Meinungs- und Kommunikationsfreiheit in wissenschaftlichen Fachzeitschriften zu schreiben. Damit verbinden wir die Hoffnung, eine für unser Fach wichtige normative Debatte zu eröffnen.

Funding Open Access funding enabled and organized by Projekt DEAL.

Open Access Dieser Artikel wird unter der Creative Commons Namensnennung 4.0 International Lizenz veröffentlicht, welche die Nutzung, Vervielfältigung, Bearbeitung, Verbreitung und Wiedergabe in jeglichem Medium und Format erlaubt, sofern Sie den/die ursprünglichen Autor(en) und die Quelle ordnungsgemäß nennen, einen Link zur Creative Commons Lizenz beifügen und angeben, ob Änderungen vorgenommen wurden.

Die in diesem Artikel enthaltenen Bilder und sonstiges Drittmaterial unterliegen ebenfalls der genannten Creative Commons Lizenz, sofern sich aus der Abbildungslegende nichts anderes ergibt. Sofern das betref- 
fende Material nicht unter der genannten Creative Commons Lizenz steht und die betreffende Handlung nicht nach gesetzlichen Vorschriften erlaubt ist, ist für die oben aufgeführten Weiterverwendungen des Materials die Einwilligung des jeweiligen Rechteinhabers einzuholen.

Weitere Details zur Lizenz entnehmen Sie bitte der Lizenzinformation auf http://creativecommons.org/ licenses/by/4.0/deed.de. 